\title{
A Unique Case of Isolated Dehydroepiandrosterone-Sulfate Secreting Adrenocortical Carcinoma: A Case Report with Review of the Literature
}

\author{
Vijaya Ganta, MD*; Gail Nunlee-Bland, MD; and Terrence Fullum, MD; \\ Division of Endocrinology, Diabetes and Metabolism, Howard University Hospital, \\ NW Washington DC, USA \\ *Corresponding Author: Vijaya Ganta, MD, Division of Endocrinology, Diabetes and \\ Metabolism, Howard University Hospital, NW Washington DC, USA.
}

Received: September 25, 2021

Published: October 19, 2021

(C) All rights are reserved by Vijaya Ganta,

MD., et al.

\begin{abstract}
Objective: Our objective is to report a case of isolated dehydroepiandrosterone-sulfate (DHEA-S) secreting adrenocortical carcinoma.

Case Presentation: A 52-year-old African American woman with a medical history of asthma for more than 10 years presented with shortness of breath, chest tightness, and cough. A diagnosis of acute asthma exacerbation with pleuritic chest pain was made, and a CT scan of the chest revealed an incidental left adrenal mass. The patient denied hyperandrogenic symptoms like deepening of the voice, acne, and excessive hair growth. The physical examination was normal, and there was no clitoromegaly. The endocrine workup was significant for increased DHEA-S $766 \mathrm{mcg} / \mathrm{dl}$ (15-170 mcg/dl), and the repeat DHEA-S was $829 \mathrm{mcg} / \mathrm{dl}$. All other adrenal hormone levels were within normal limits. The CT scan of the abdomen and pelvis without contrast revealed a $5.5 \mathrm{~cm}$ left adrenal mass with Hounsfield units of 23. An open left adrenalectomy was done. Histopathology was reported as marked and diffuse nuclear atypia, necrosis, and atypical mitotic figures. The tumor was strongly positive for inhibin, synaptophysin, and melan-A. The Ki-67 immunostain showed $20-25 \%$ positive staining.
\end{abstract}

Discussion: Adrenocortical carcinoma (ACC) is a rare adrenal cortex malignancy. Both size more than $4 \mathrm{~cm}$ and radiographic appearance are significant predictors of malignancy. An isolated DHEA-S secreting ACC patient may have a DHEA-S level more than 3 times the normal upper limit.

Conclusion: Isolated DHEA-S secreting adrenocortical carcinoma patients may present without hyperandrogenic features like hirsutism and virilization.

\section{Abbreviations}

DHEA: Dehydroepiandrosterone; DHEA-S: Dehydroepiandrosterone-sulfate; ACC: Adrenocortical Carcinoma; ACTH: Adrenocorticotropic Hormone; DHT: Dihydrotestosterone; CT: Computed Tomography; FSH: Follicle-Stimulating Hormone; LH: Luteinizing Hormone

\section{Introduction}

Adrenocortical carcinoma is a rare adrenal cortex malignancy. It presents in as few as 1-2 per million of the population in the United States [1]. It accounts for $0.2 \%$ of annual deaths in the United States [2]. The tumors which are functional in nature often present with a clinical picture of Cushing's syndrome or a mixed hormonal pat- 
tern. A smaller subset of patients present with purely androgen hypersecretion, which is more common in women than men $[3,4]$. The most commonly affected age group is adults in their fourth and fifth decades of life. However, the distribution is bimodal, also presenting in childhood $[2,5]$. Patients with functioning tumors account for approximately $60 \%$, while patients with nonfunctioning tumors account for $40 \%$ of cases presenting with mass effect [6].

\section{Case Presentation}

A 52-year-old African American woman with a medical history of type 2 diabetes, obesity, status post-Roux-en-Y gastric bypass, hypertension, and asthma for more than 10 years, presented with shortness of breath, chest tightness, and cough. A diagnosis of acute asthma exacerbation with pleuritic chest pain was made and a CT scan of the chest revealed an incidental left adrenal mass. The Patient denied hyperandrogenic symptoms like deepening of the voice, acne, excessive hair growth on the face, back, and chest. The Physical examination was normal, and there was no clitoromegaly. The endocrine workup was significant for an elevated DHEA-S level of $766 \mathrm{mcg} / \mathrm{dl}$ (15-170 mcg/dl), and the repeat DHEA-S was 829 $\mathrm{mcg} / \mathrm{dl}$. All other adrenal hormone levels are included below.

ACTH- $6 \mathrm{pg} / \mathrm{ml}$ (6-50); Androstenedione- $25 \mathrm{ng} / \mathrm{dl}$ (20-75); DHEA- $3 \mathrm{ng} / \mathrm{ml}(<6)$; Total Testosterone- $7 \mathrm{ng} / \mathrm{dl}(2-45)$; free Testosterone- $2.1 \mathrm{pg} / \mathrm{ml}$ (0.1-6.4); Estradiol (post-menopausal)$16 \mathrm{pg} / \mathrm{ml} \mathrm{(<31);} \mathrm{FSH} \mathrm{(post-menopausal)-} 78.3 \mathrm{IU} / \mathrm{L}(>30)$; LH- 22.5 IU/L (1.4-7.7); Aldosterone- 6 ng/dl (3-16); Plasma Renin Activity- $1.98 \mathrm{ng} / \mathrm{ml} / \mathrm{hr}$ (0.25-5.82); 24 hour urinary: Free Cortisol- $43.8 \mathrm{mcg} / 24 \mathrm{hrs}$ (4.0-50); Epinephrine- $3 \mathrm{mcg} / 24 \mathrm{hr}$ (2-24) Norepinephrine- $47 \mathrm{mcg} / 24 \mathrm{hr}$ (15-100); Dopamine- 236 mcg/24hr (52-480); Metanephrines- 516 mcg/24hr (224-832).

All Adrenal hormone levels, except DHEA-S, were within normal limits. FSH and LH concentrations were appropriately elevated for the post-menopausal state. The CT scan of the abdomen and pelvis without contrast revealed a $5.5 \mathrm{~cm}$ left adrenal mass with Hounsfield units of 23.

The patient underwent open left adrenalectomy based on the size and imaging phenotype. The adrenal mass was well encapsulated, and the patient tolerated the procedure without complications.

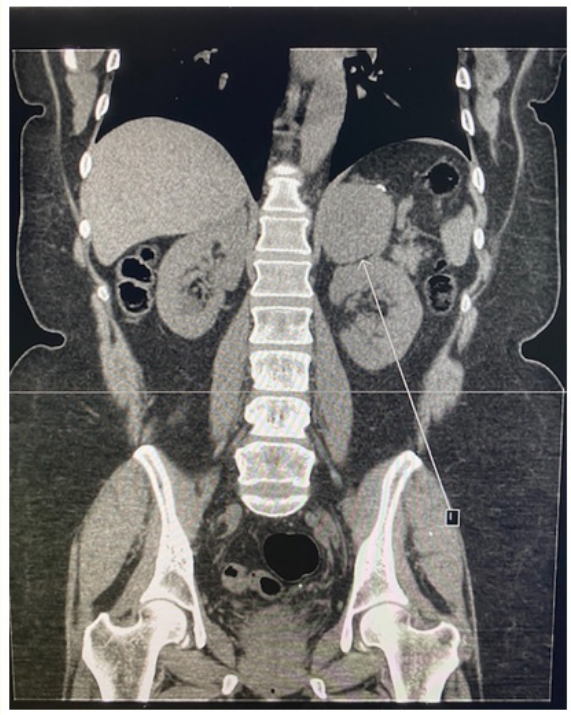

Figure 1: CT scan of abdomen and pelvis without contrast, coronal view revealed $5.5 \mathrm{~cm}$ left adrenal mass.

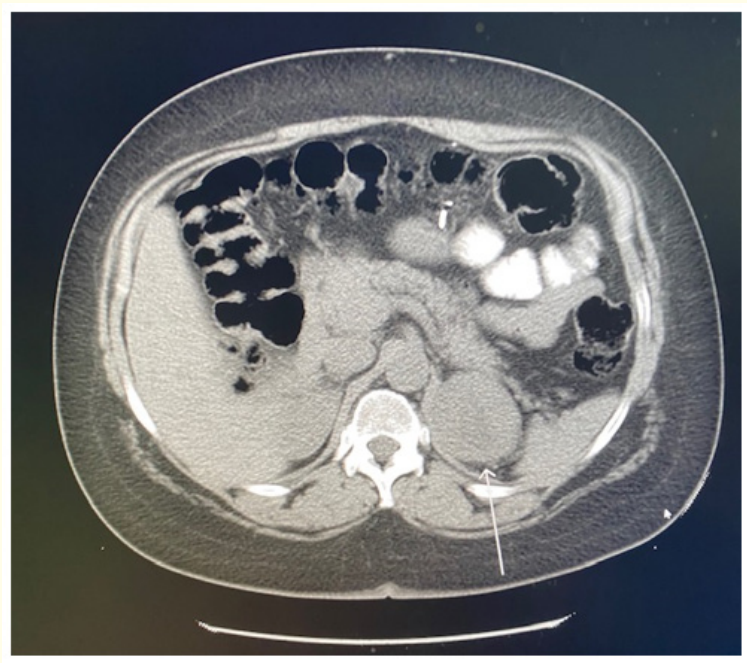

Figure 2: CT scan of abdomen and pelvis without contrast, transverse view revealed $5.5 \mathrm{~cm}$ left adrenal mass with Hounsfield units of 23. 
Pathology revealed grossly, the specimen consisted of a 102gram mass measuring $6.5 \times 5.5 \times 5.2 \mathrm{~cm}$. Sectioning and inking revealed $6.6 \times 5.2 \times 5.1 \mathrm{~cm}$ tan-brown mass with focal areas of necrosis in the center of the lesion. Microscopically, hematoxylin and eosin staining showed marked and diffuse nuclear atypia, necrosis, and atypical mitotic figures. Immunohistochemical studies revealed that the tumor cells were strongly positive for inhibin, synaptophysin, and melan-A, which are the markers of adrenocortical carcinoma. It was weakly positive for S100 and negative for HMB-45. The Ki-67 immunostain showed approximately $20-25 \%$ positive staining. P53 staining showed less than $10 \%$ positive cells. The gross and immunohistochemical findings support the diagnosis of adrenocortical carcinoma.

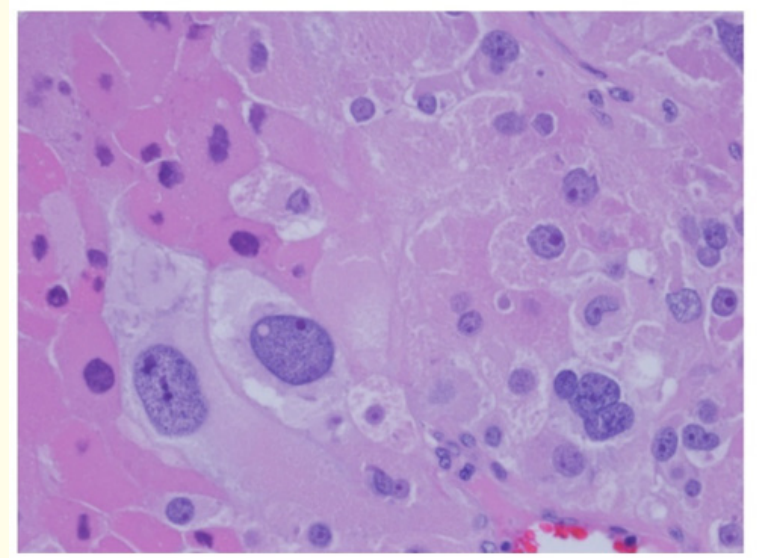

Figure 3: Left adrenal tumor H\&E. The tumor showed marked nuclear pleomorphism with diffuse nuclear atypia.

At one month post-adrenalectomy, serum DHEA-S concentration was less than $15 \mathrm{mcg} / \mathrm{dl}$ and remained normal. The patient was started on mitotane to prevent recurrence and was on treatment for 3 years and stopped. The patient is doing well and is being followed up with a yearly DHEA-S level that remained less than 15 $\mathrm{mcg} / \mathrm{dl}$ without recurrence, and a CT scan of the abdomen reported as no recurrence.

\section{Discussion}

Our patient presented with symptoms of acute asthma exacerbation. A CT scan of the chest revealed an incidental $5.5 \mathrm{~cm}$

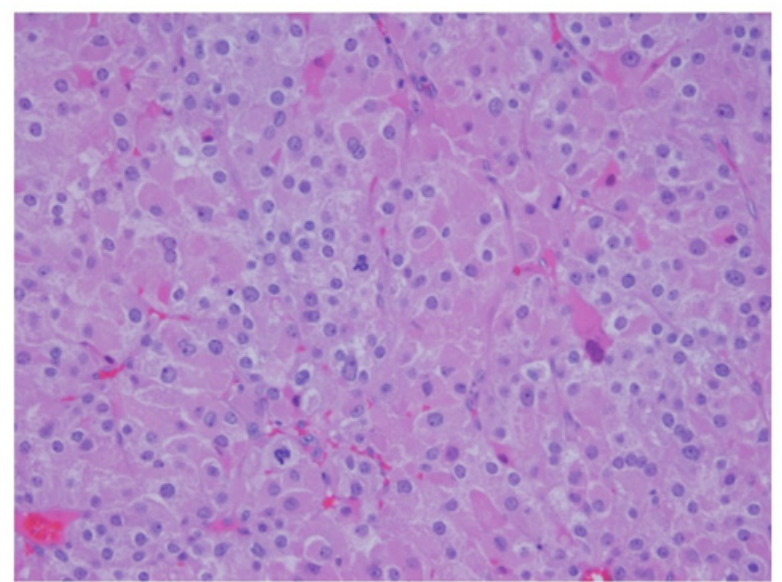

Figure 4: There was a high mitotic rate with atypical mitotic figures.

left adrenal mass and DHEA-S was more than 3 times the normal upper limit. All other adrenal hormone levels were within normal limits. The patient underwent a left adrenalectomy. Histopathology was reported as diffuse nuclear atypia and atypical mitotic figures. The tumor cells were strongly positive for inhibin, synaptophysin, and melan-A. So, a diagnosis of isolated DHEA-S secreting ACC was made.

ACC is found in approximately $4.7 \%$ of incidentally discovered adrenal masses, and 2.5\% are metastatic upon presentation [7]. Both size and radiographic appearance are significant predictors of malignancy. Tumor size greater than $4 \mathrm{~cm}$ has reported a $90 \%$ sensitivity of predicting carcinoma but relatively low specificity of $24 \%$ [8]. According to the National Institute of Health consensus statement, tumors larger than $6 \mathrm{~cm}$ are highly suspicious for malignancy and should be removed [9]. Adrenocortical carcinoma has a relatively poor prognosis with an average 5-year mortality of $75-90 \%$ [10], and shows signs of relapse or metastasis in $70-85 \%$ [11].

DHEA-S, DHEA, and androstenedione may be considered prohormones, requiring conversion to testosterone or DHT to express androgenic effects such as hirsutism and virilization. DHEA-S level above $600 \mathrm{mcg} / \mathrm{dl}$ or more than 3 times the normal 
upper limit of $(15-170 \mathrm{mcg} / \mathrm{dl})$ is diagnostic of adrenocortical carcinoma [12]. DHEA-S secreting adrenocortical carcinomas lose the ability to produce testosterone may be due to relative deficiency of 3-beta-hydroxysteroid dehydrogenase activity. So, their testosterone level will be normal, and patients may not present with hirsutism or virilization. However, adrenocortical adenomas produce excess testosterone, and patients present with androgenic effects.

In patients with ACC, tumor cells will be strongly positive for tumor markers such as inhibin, synaptophysin, and melan-A. ACC is treated with complete surgical resection in patients who are surgical candidates. Mitotane therapy is used for unresectable disease and disease recurrence. Radiation therapy is used for metastatic tumors.

Our patient presented with several predictive factors of malignant potential including tumor size and radiographic appearance, pathology of the mass showing high mitotic rate, and tumor necrosis has been related to a poor prognosis $[13,14]$. However, our patient is doing well and has been cured of ACC without recurrence through surgery and mitotane. The DHEA-S level remains less than $15 \mathrm{mcg} / \mathrm{dl}$.

\section{Conclusion}

Isolated DHEA-S secreting adrenocortical carcinoma patients may present without hyperandrogenic features such as hirsutism and virilization.

\section{Consent for Publication}

Written informed consent to publish this case report and accompanying images has been obtained from the person described in the case.

\section{Conflicts of Interest}

The authors declare that they have no conflicts of interest.

\section{Sources of Support}

There are no sources of support.

\section{Acknowledgements}

A special thanks to Dr. Bonnie Davis and Dr. Hassan Nabhani for radiology images and Dr. Lekidelu Taddesse-Heath for pathology slides.

\section{Bibliography}

1. Bodie B., et al. "The Cleveland Clinic experience with adrenal cortical carcinoma". The Journal of Urology 141.2 (1989): 257260.

2. Wajchenberg Bernardon Leo., et al. "Adrenocortical carcinoma". Cancer 88.4 (2000): 711-736.

3. Luton Jean-Pierre., et al. "Clinical features of adrenocortical carcinoma, prognostic factors, and the effect of mitotane therapy". New England Journal of Medicine 322.17 (1990): 1195-1201.

4. Allolio Bruno and Martin Fassnacht. "Adrenocortical carcinoma: clinical update". Journal of Clinical Endocrinology and Metabolism 91.6 (2006): 2027-2037.

5. Fassnacht Martin and Bruno Allolio. "Clinical management of adrenocortical carcinoma". Best Practice and Research Clinical Endocrinology and Metabolism 23.2 (2009): 273-289.

6. Ng Linda and John M Libertino. "Adrenocortical carcinoma: diagnosis, evaluation and treatment". The Journal of Urology 169.1 (2003): 5-11.

7. Young Jr and William F. "Management approaches to adrenal incidentalomas: a view from Rochester, Minnesota". Endocrinology and metabolism clinics of North America 29.1 (2000): 159-185.

8. Angeli A., et al. "Adrenal incidentaloma: an overview of clinical and epidemiological data from the National Italian Study Group". Hormone Research in Paediatrics 47.4-6 (1997): 279283.

9. Grumbach MM., et al. "Management of the clinically inapparent adrenal mass ("incidentaloma")". Annals of Internal Medicine 138 (2003): 424-429.

10. Balasubramanian Sanjeeve and Tito Fojo. "Practical considerations in the evaluation and management of adrenocortical cancer". Seminars in Oncology 37.6 (2010): 619626.

11. Veytsman Irina., et al. "Management of endocrine manifestations and the use of mitotane as a chemotherapeutic 
agent for adrenocortical carcinoma". Journal of Clinical Oncology

27.27 (2009): 4619-4629.

12. Fassnacht M., et al. "Adrenal tumors: how to establish malignancy?". Journal of Endocrinological Investigation 27.4 (2004): 387-399.

13. Stojadinovic Alexander., et al. "Adrenocortical carcinoma: clinical, morphologic, and molecular characterization". Journal of Clinical Oncology 20.4 (2002): 941-950.

14. Wachenfeld Christoph., et al. "Discerning malignancy in adrenocortical tumors: are molecular markers useful?" European Journal of Endocrinology 145.3 (2001): 335-341.

Volume 5 Issue 11 November 2021

(c) All rights are reserved by Vijaya Ganta, MD., et al. 